\title{
ounersis
}

\section{Choosing a second-line therapy after metformin in type 2 diabetes mellitus}

T wo multinational, randomized, open-label studies published in The Lancet shed light on the pros and cons of different second-line agents for the treatment of patients with type 2 diabetes mellitus (T2DM) inadequately controlled by metformin.

Metformin is an established firstline therapy for T2DM. As T2DM is a progressive disease, when metformin stops providing adequate glycaemic control treatment escalation is required. However, insufficient long-term, headto-head comparison data exist to help clinicians make an evidence-based therapeutic decision at this stage. Secondline agents for T2DM therapy include other oral agents or injectable agents, such as exenatide or insulin.

In the first study, the European Exenatide (EUREXA) trial, the efficacy and the long-term sustainability of the GLP-1 receptor agonist exenatide were compared with those of the sulphonylurea glimepiride in patients with overweight or obesity who had T2DM not adequately controlled by metformin monotherapy ( $\mathrm{HbA}_{1 \mathrm{c}}$ levels $\geq 6.5 \%$ and $\leq 9 \%$ ).

"At the time the study was designed, in 2005, data from basic research and animal studies suggested that exenatide might slow the progression of T2DM and it was already known that sulphonylureas lead to secondary treatment failure," comments lead author Baptist Gallwitz of EberhardKarls-University Tübingen, Germany.

The researchers randomly assigned 1,029 patients to receive exenatide twice daily or glimepiride once daily as add-on to metformin for up to 4.5 years. The primary outcome of the study was time to inadequate glycaemic control, which was defined as an $\mathrm{HbA}_{1 c}$ level of $>9 \%$ after 3 months of second-line treatment or $>7 \%$ at two consecutive visits after 6 months of second-line treatment.

Exenatide was superior to glimepiride in preventing inadequate glycaemic control: 203 (41\%) of patients in the exenatide group had treatment failure compared with 262 (54\%) in the glimepiride group. An $\mathrm{HbA}_{1 \mathrm{c}}$ level of $<7 \%$ was achieved by more patients in the exenatide than in the glimepiride group. Moreover, fewer patients in the exenatide group than in the glimepiride group reported hypoglycaemic episodes. Patients receiving exenatide also lost weight (mean $3.32 \mathrm{~kg}$ ), whereas those in the glimepiride group gained weight (mean $1.15 \mathrm{~kg}$ ). Nevertheless, discontinuation of therapy owing to adverse effects (mainly nausea) was higher in the exenatide group than in the glimepiride group in the first 6 months of treatment.

"The findings support the concept that GLP-1 receptor agonists have an important place in second-line therapy of T2DM after metformin failure," says Gallwitz.

The second study was a head-to-head comparison of insulin glargine and the DPP-4 inhibitor sitagliptin in 515 patients with T2DM and inadequate glycaemic control ( $\mathrm{HbA}_{1 \mathrm{c}}$ levels 7-11\%) who were receiving metformin.

"Insulin has usually been left as a last resource when all the oral glucoselowering drugs have failed, but in the past few years insulin has been proposed as a second-line therapy option," explains lead researcher Pablo Aschner of Pontificia Universidad Javeriana, Colombia. "Several trials have compared DPP-4 inhibitors with other oral glucose-lowering drugs added on to metformin but none has compared DPP-4 inhibitors with insulin."

The efficacy of insulin glargine to reduce $\mathrm{HbA}_{1 \mathrm{c}}$ levels during the 24 -week study was superior to that of sitagliptin (mean difference in $\mathrm{HbA}_{1 \mathrm{c}}$ levels -0.59\%). Patients treated with insulin glargine were 1.6 times more likely than those treated with sitagliptin to achieve an $\mathrm{HbA}_{1 \mathrm{c}}$ level $<7 \%$. Nevertheless, rates of hypoglycaemia per patient-year were eight times higher with insulin glargine than with sitagliptin,

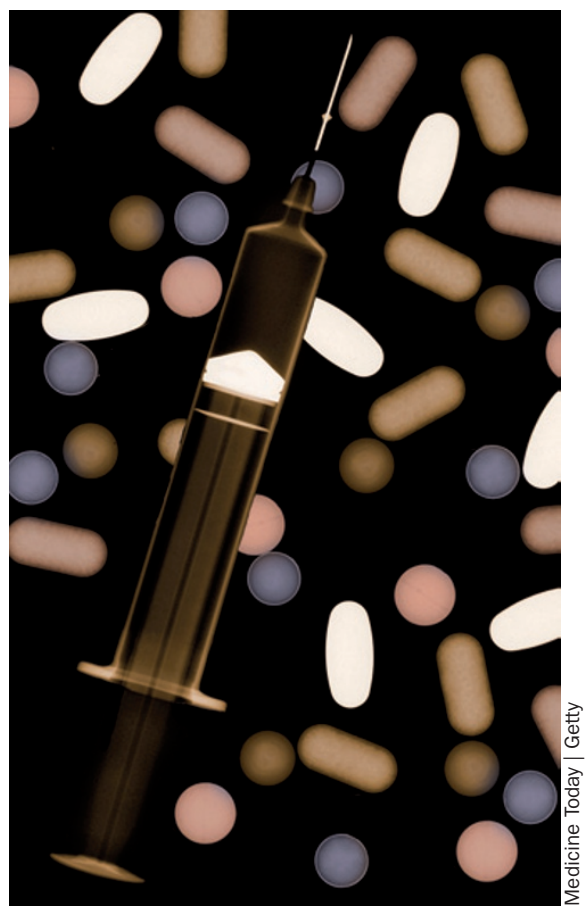

although severe events were rare in both groups. Patients treated with insulin glargine had only a slight increase in weight, whereas those treated with sitagliptin lost a small amount of weight.

"The results of this study support the option of introducing basal insulin (glargine) in patients with T2DM inadequately controlled by metformin, with the potential for long-term benefits arising from the achievement of optimum glycaemic control early in the course of the disease," comments Aschner.

The insights of these two studies provide stepping stones on the long path towards individualised medicine for patients with T2DM.

Carol Wilson

Original articles Gallwitz, B. et al. Exenatide twice daily versus glimepiride for prevention of glycaemic deterioration in patients with type 2 diabetes with metformin failure (EUREXA): an open-label, randomised controlled trial. Lancet doi:10.1016/S0140-6736 | Aschner, P. et al. Insulin glargine versus sitagliptin in insulin-naive patients with type 2 diabetes mellitus uncontrolled on metformin (EASIE): a multicentre, randomised open-label trial. Lancet doi:10.1016/S0140-6736 